\title{
Papillary thyroid cancer in a struma ovarii: a report of a rare case
}

\author{
Eleonora Monti, ${ }^{1}$ Lorenzo Mortara, ${ }^{1}$ Simonetta Zupo, ${ }^{2}$ Mariella Dono, ${ }^{2}$ \\ Francesco Minuto, ${ }^{1}$ Mauro Truini, ${ }^{2}$ Mehrdad Naseri, ${ }^{3}$ Massimo Giusti ${ }^{1}$
}

${ }^{1}$ Department of Internal Medicine, Endocrinology Unit, ${ }^{2}$ Department of Pathology, IRCCS IST Azienda Ospedaliera Universitaria "San Martino"; ${ }^{3}$ Department of Nuclear Medicine, E.O. Ospedali Galliera; Genoa; Italy

\begin{abstract}
After removal of an ovarian mass in a 43-year-old woman, a struma ovarii was diagnosed. Within this teratoma, a papillary thyroid cancer was found. The tumor was negative for BRAF, NRAS, KRAS, PIK3CA and c-KIT mutations on molecular analysis. Thyroid function and morphology were normal. Thyroidectomy, L-T4 TSH-suppressive therapy and rhTSH-induced radioiodine ablation were performed. So far, the follow-up has been favorable. This is the first case of thyroid cancer in a struma ovarii in which mutations of PIK3CA exons 9 and 20, and c-KIT exons 9, 11 and 13 have been evaluated and the third in which ablation has been performed under rhTSH. The prognosis of patients with thyroid cancer in a struma ovarii is generally poor. In our patient, as in those who undergo ablative radioactive iodine therapy, this was not the case.
\end{abstract}

Key words: Papillary carcinoma, rhTSH, Struma ovarii, Teratoma

\section{INTRODUCTION}

A rare ovarian teratoma consisting mainly of thyroid tissue, struma ovarii accounts for up to $3 \%$ of all ovarian tumors. While malignancy occurs in $5-10 \%$ of cases, ${ }^{1}$ only about $5 \%$ of malignant strumae metastasize. ${ }^{2}$ Benign and malignant strumae manifest different behaviors, in that the former generally exhibit iodine uptake and may even secrete significant amounts of thyroid hormones, ${ }^{3}$ while the latter display poor iodine uptake and infrequently secrete thyroid hormones.

\footnotetext{
Address for correspondence:

Eleonora Monti, MD, Dipartimento di Medicina Interna, Cattedra di Endocrinologia, University of Genova, Italy, Viale Benedetto XV, ${ }^{\circ} 6$, I-16132 Genoa, Italy

Fax: +39-010 3538977, E-mail: eleonora.monti87@gmail.com

Received: 02-12-2013, Accepted: 27-03-2014
}

The therapy of this tumor has not been well defined. ${ }^{2}$ However, in the case of malignant strumae, treatment will normally involve removal of the primary ovarian tumor and the thyroid gland, followed by ${ }^{131} \mathrm{I}$ therapy, which can then be administered under hypothyroid conditions. In thyroid carcinoma metastases, iodine uptake is usually low, but can be increased by TSH stimulation in a hypothyroid state before ${ }^{131}$ I treatment. Although the most effective way of achieving a cytotoxic concentration of ${ }^{131}$ I per gram of tumor has not yet been established, stimulation either with endogenous TSH after the administration of an anti-thyroid drug or with recombinant human TSH (rhTSH), in order to obtain optimal TSH levels for ${ }^{131}$ I scanning and therapy, may be potentially efficacious strategies. ${ }^{2}$ 


\section{DESCRIPTION OF THE PATIENT}

A 43-year-old woman was referred to our clinic in January 2013 after video-laparoscopic surgery (November, 2012) and histological findings of mature cystic triphasic teratoma and a significant thyroid component of carcinomatous transformation (follicular variant of papillary carcinoma). The patient had been followed up for about two years for a left ovarian cyst and underwent surgery because of increased cyst volume, a subjective feeling of weight in the left hypochondrium and urinary disorders. Her BMI was $18 \mathrm{~kg} / \mathrm{m}^{2}$ and her blood pressure was $100 / 60 \mathrm{mmHg}$. At the time of our observation she was euthyroid (Table 1) and anti-thyroglobulin (Tg) antibodies ( $\mathrm{TgAb}$ ) were negative. The only data available before the left oophorectomy dated back to 2010 and showed a euthyroid condition; moreover, her anamnesis revealed no sign of hyperthyroidism. The remaining blood parameters were normal $(\mathrm{Hb} 12.8$ $\mathrm{mg} / \mathrm{dl}, \mathrm{MCV} 86 \mathrm{fl}$, Hct 39.6\%) and ultrasound of the neck showed a thyroid in place, within normal limits in all dimensions (AP diameter $13 \mathrm{~mm}$ right lobe, left lobe $10 \mathrm{~mm}$ ), homogeneous, with no evidence of nodular formations. Staging was performed by means of total-body PET ${ }^{18}$ F-FDG (with a Siemens somatom sensation 16 biograph, Rome, Italy) which showed no proliferative-hypermetabolic areas in the zones included in the anatomical scan planes. Subsequently, the patient underwent prophylactic total thyroidectomy, after which thyroid parenchyma was detected (normal) and treatment with oral L-T4 replacement ( $75 \mu \mathrm{g} /$ day) was administered. There was no ascites or pleural effusion. Genetic analysis for some common point mutations in thyroid cancers was performed on the tumor tissue: BRAF (codons
598, 599, 600), NRAS (codon 61), KRAS (codons 12 and 13), PIK3CA (exons 9 and 20) and c-KIT (exons $9,11,13)$ all proved unmutated. Thereafter, the patient underwent treatment with ablative radio-iodine (RAI; $3700 \mathrm{MBq}$ ) while on a low-iodine diet (urinary iodine $27 \mu \mathrm{g} / \mathrm{L}$ ); rhTSH (Genzyme, Modena, Italy) was administered at a dosage of $0.45 \mathrm{mg} /$ day i.m. for 4 days, the last dose being given 24 hours before the administration of radioiodine. Whole-body scan (rxWBS) was performed by means of a Philips Forte (Rome, Italy) gamma camera 48 (Figures. 1 and 2), 72 and 168 (Figure 3 ) hours after administration of ${ }^{131} \mathrm{I}$. RxWBS showed a zone of hyperaccumulation of the tracer at the base of the neck, attributable to residual thyroid iodine absorption, without any other areas of significant hyperfixation in the remaining body segments. In particular, the SPECT acquisitions at 168 hours did not identify areas of pathological hyperfixation in the pelvis (Figure 3). During the scan, TSH was $>100 \mathrm{mIU} / \mathrm{L}$, Tg was undetectable $(<0.4 \mu \mathrm{g} / \mathrm{L})$ and $\mathrm{TgAb}$ was negative $(14 \mathrm{IU} / \mathrm{mL})$. On day 7 after the discontinuation of rhTSH, Tg was $0.97 \mu \mathrm{g} / \mathrm{L}$ and TSH $11.3 \mathrm{mIU} / \mathrm{L}$; the oral L-T4 dosage was increased

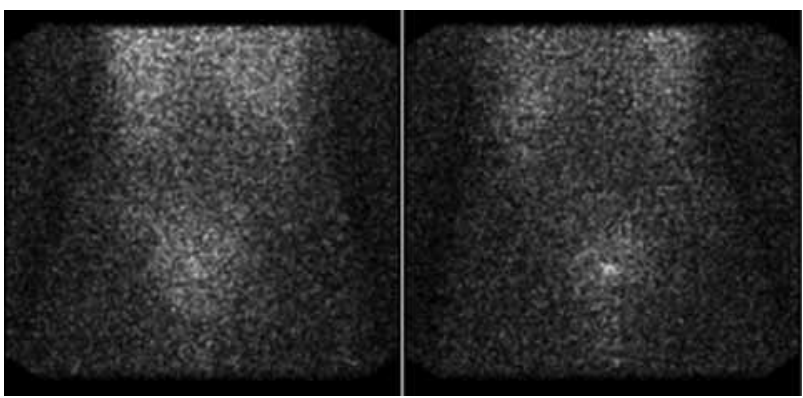

Figure 1. Anterior (a) and posterior (b) views of the rxWBS performed 48 hours after iodine administration.

Table 1. Historical and current data on the patient's thyroid function

\begin{tabular}{lcccccc}
\hline & $\begin{array}{c}\text { July } \\
\mathbf{2 0 1 0}\end{array}$ & $\begin{array}{c}\text { January } \\
\mathbf{2 0 1 3}\end{array}$ & $\begin{array}{c}\text { March 2013 } \\
\text { (after thyroidectomy) }\end{array}$ & $\begin{array}{c}\text { April 2013 } \\
\text { (before RAI) }\end{array}$ & $\begin{array}{c}\text { April 2013 } \\
\text { (under rhTSH, during RAI) }\end{array}$ & $\begin{array}{c}\text { September } \\
\mathbf{2 0 1 3}\end{array}$ \\
\hline $\mathrm{f}-\mathrm{T} 3(\mathrm{pmol} / \mathrm{L})$ & 4.45 & 5.2 & 4.68 & 3.6 & 3.375 & $\mathrm{~N} / \mathrm{A}$ \\
$\mathrm{f}-\mathrm{T} 4(\mathrm{pmol} / \mathrm{L})$ & 15.4 & 15 & 9.4 & 15.4 & 16.8 & 16.7 \\
$\mathrm{TSH}(\mathrm{mIU} / \mathrm{L})$ & 1.36 & 1.68 & 0.72 & 8.7 & $>100$ & 2.18 \\
$\mathrm{Tg}(\mu \mathrm{g} / \mathrm{L})$ & $\mathrm{N} / \mathrm{A}$ & 6.8 & 9.89 & 0.4 & 0.1 & $<0.5$ \\
$\mathrm{TgAb}(\mathrm{IU} / \mathrm{mL})$ & $\mathrm{N} / \mathrm{A}$ & 35 & 26 & 12 & $\mathrm{~N} / \mathrm{A}$ & 15 \\
\hline
\end{tabular}

Abbreviations: N/A: not available

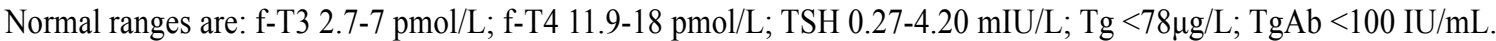




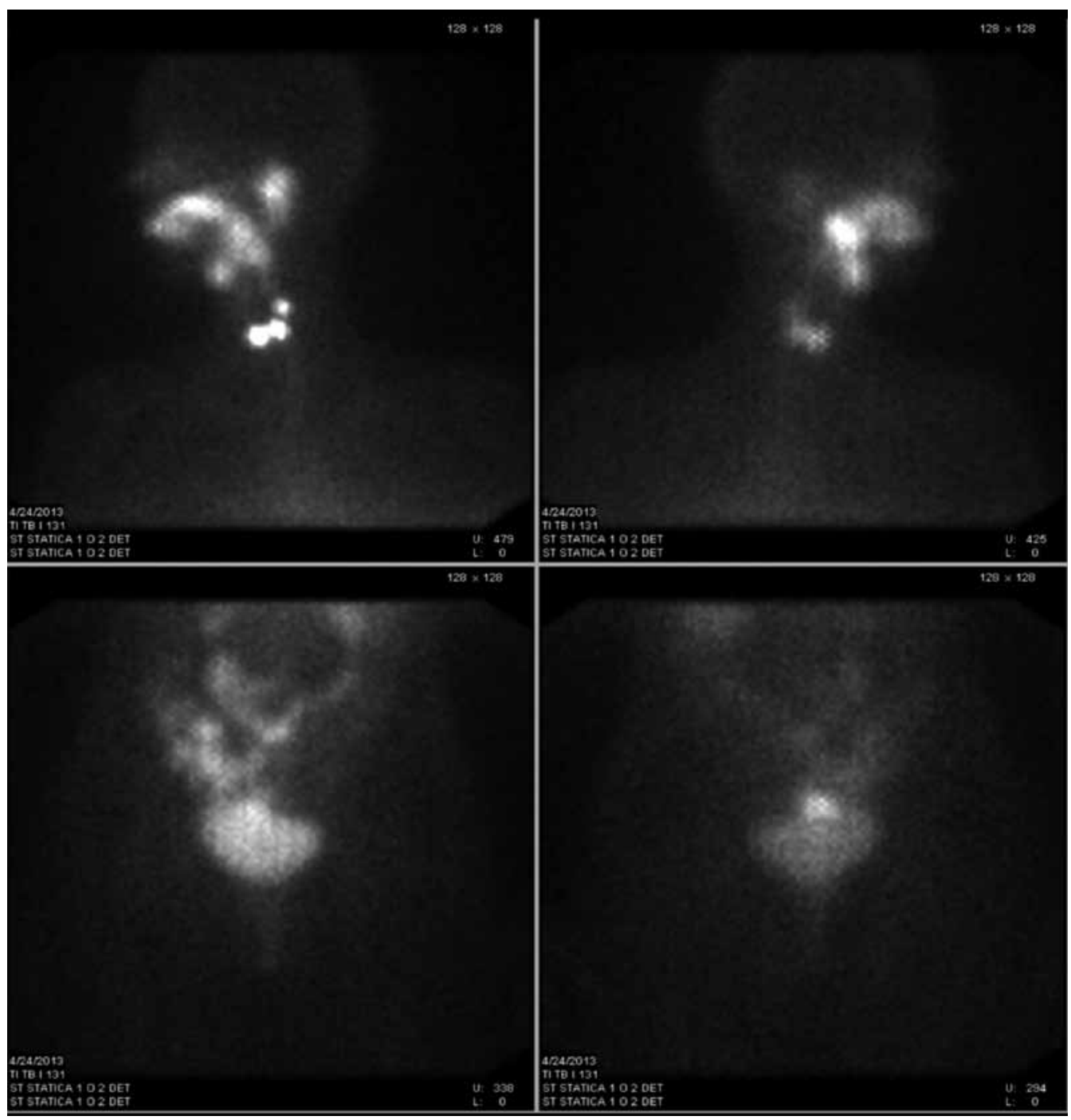

Figure 2. Magnification views of neck (upper panels) and pelvic (lower panels) scans performed 48 hours after iodine administration.

to $100 \mu \mathrm{g} /$ day to achieve long-term suppression of TSH. Menstrual flows were consistently regular. No data on Ca-125 levels were available.

\section{DISCUSSION}

Thyroid cancer in a struma ovarii is rare, arising in approximately $5-10 \%$ of all struma ovarii and accounting for $3 \%$ of germ cell ovarian cancers. ${ }^{4}$ Struma ovarii can coexist with normal thyroid or with numerous thyroid abnormalities: hyperplasia, thyroiditis, hyperthyroidism, adenoma or carcinoma. ${ }^{4}$ The criteria for the diagnosis of malignancy in struma ovarii are debatable. Pardo-Mindan et al noted that nuclear alterations alone were not sufficient diagnostic criteria, given the fact that atypical cells are frequent in noncancerous lesions. ${ }^{5}$ These authors emphasized the importance of excluding metastasis from a neck thyroid cancer before rendering a diagnosis of malignant struma ovarii. ${ }^{5}$ They also claimed that the presence of invasion of the capsule or peritoneal sites of disease should be required for the diagnosis of malignancy. ${ }^{5}$ None of these features was present on histological examination of our patient. The Tg immune-staining of ovarian tissue is also extremely important as a diagnostic tool for confirming the thyroid nature of an ovarian teratoma. ${ }^{1}$ Despite its rarity and its relatively good prognosis, the treatment 


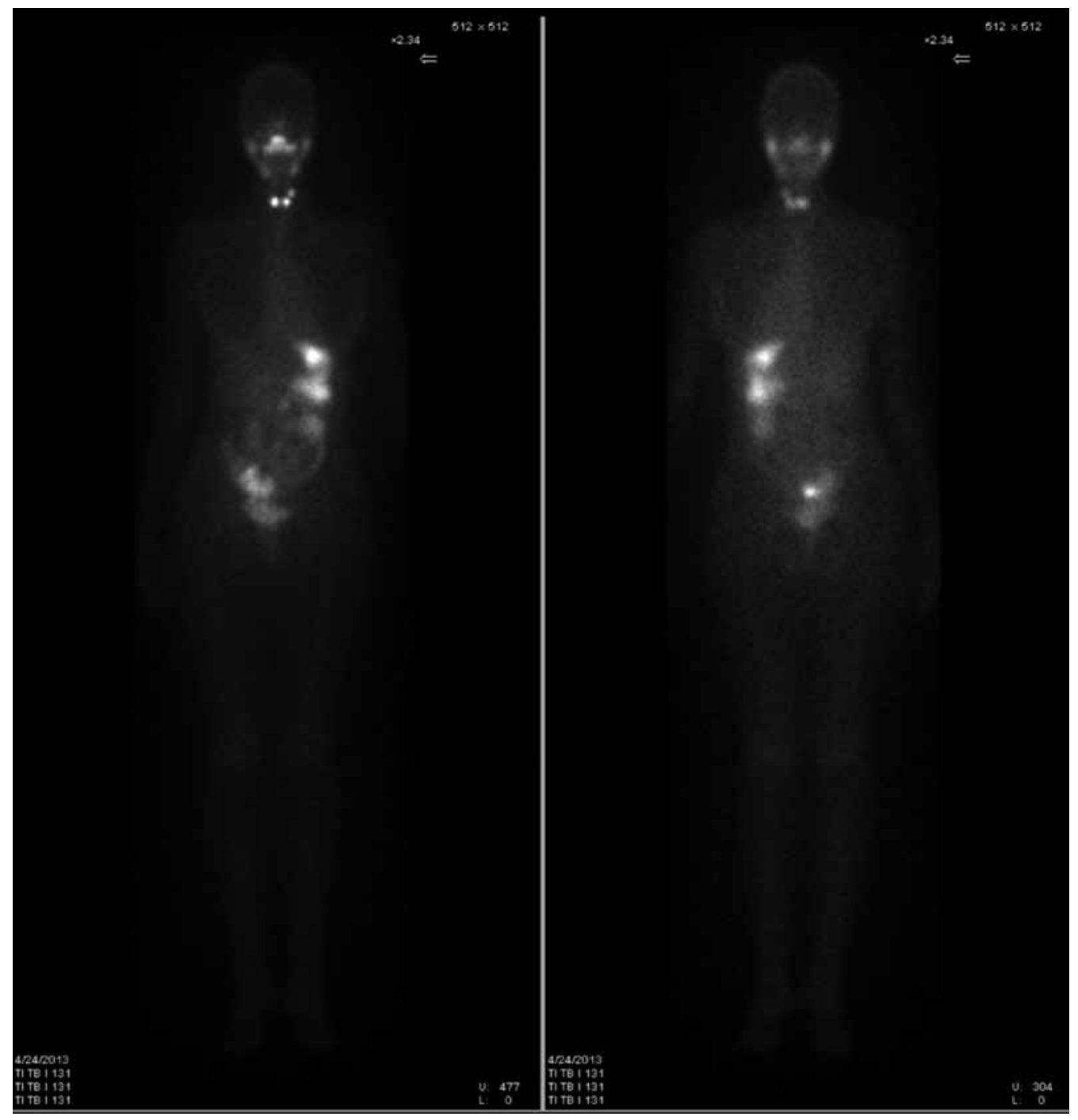

Figure 3. Anterior (a) and posterior (b) magnification of the pelvic scan performed 168 hours after iodine administration.

of ovarian teratoma has long been debated. The therapeutic protocol is similar to that implemented for thyroid cancer, including surgical removal of the thyroid gland and the ovarian mass, RAI ablation and, subsequently, TSH-suppressive L-T4 therapy. ${ }^{6}$ In order to allow ablative treatment with ${ }^{131} \mathrm{I}$ to be carried out, thyroidectomy is necessary, as is subsequent follow-up. Other therapeutic modalities have also been used, such as intraperitoneal chromic P32 phosphate or radioactive colloidal gold and external beam radiation. However, we believe that RAI must constitute the first line of therapy after surgical debulking. ${ }^{1}$ In our case, the patient had no metastases on diagnosis and was not hyperthyroid. As shown by the literature data, only a small portion of struma ovarii metastasize; however, if thyrotoxicosis is present, distant metastases coexist in $83 \%$ of cases. ${ }^{2}$ Interestingly, the majority of metastases produce T3; this could be caused either by overproduction of $\mathrm{T} 3$ or by the increased conversion of $\mathrm{T} 4$ to $\mathrm{T} 3$ at the peripheral level. ${ }^{2}$ In our case, no data on thyroid function before the removal of the ovarian mass were available (a single sample from 2010 showed a euthyroid state); however, we never observed a condition of hyperthyroidism or the increased presence of T3 in serum. Despite the absence of metastases on diagnosis, we treated the patient with ablative RAI after thyroidectomy, since these radical procedures 
offer greater safety. Indeed, some authors recommend thyroidectomy and ${ }^{131} \mathrm{I}$ radio ablation to reduce local and distant recurrence, which display rates as high as $16-35 \% .^{7}$ De Simone et al reported that none of six patients who received adjuvant therapy with thyroidectomy and RAI after surgical resection of a struma ovarii suffered recurrence. ${ }^{8}$ By contrast, in 42 patients who were treated only surgically, without any further adjuvant therapy, Jean et al found that nine (21\%) suffered recurrence. ${ }^{7}$ In our patient, rxWBS revealed areas of enhanced ${ }^{131} \mathrm{I}$ uptake only at the base of the neck, further demonstrating the absence of metastasis and the radical nature of pelvic surgery.

With regard to genetic analyses, a KRAS mutation was reported in a 35-year-old female in whom struma ovarii (at final pathology a non-encapsulated focus of follicular variant of papillary thyroid carcinoma) was diagnosed during the first trimester of pregnancy. ${ }^{4}$ Schmidt et al found that BRAF mutations were present in 4 of 6 malignant struma ovarii (4 follicular variants, 1 classic and 1 metastasis of a classic) and in none of 9 benign struma ovarii. These BRAF mutations included V600E, K601E and a novel deletion/ substitution TV599-600M. Neither malignant nor benign struma ovarii contained alterations in NRAS, KRAS or RET. ${ }^{9}$ Moreover, no follicular carcinomas were identified in their study. ${ }^{9}$ Numerous studies of thyroid carcinomas have suggested that BRAF mutations are associated with older age, advanced disease, classical papillary histology and poorer prognosis, as indicated by disease-free and overall survival. ${ }^{10,11}$ The presence of RET/PTC rearrangement correlates with some clinical pathological features of PTC, such as younger age, tumor morphology and a higher probability of lymph node involvement. ${ }^{12}$ In our patient, the search for mutations of BRAF 598 and 599 and 600 exon 15, NRAS codon 61, KRAS codons 12 and 13, PIK3CA exons 9 and 20, and c-KIT exons 9, 11 and 13 was negative (Figure 4).

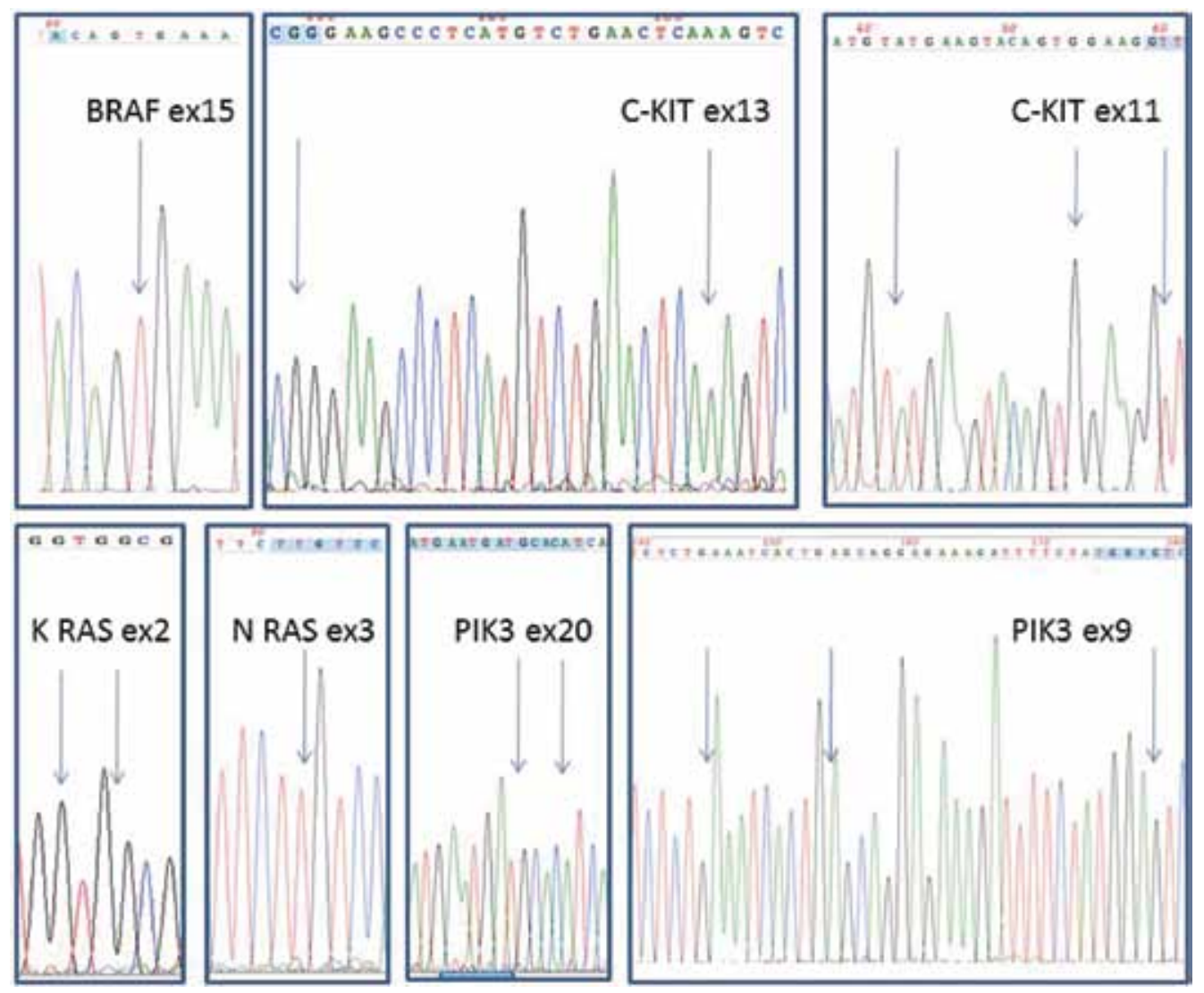

Figure 4. KRAS, NRAS, BRAF, PIK3CA, c-KIT genotypes. Sequencing electropherograms of the indicated wild-type genes are reported. Arrows indicate the codons in which hotspot mutations were described. 


\section{CONCLUSIONS}

Malignant struma ovarii is a rare disease, its prognosis is still not well known and its treatment is still debatable. Our case is interesting because, owing to the rarity of this tumor, experience is limited and therapy has not been well defined. Moreover, no metastases or clinical hyperthyroidism were found during follow-up. After total thyroidectomy and removal of the ovarian mass, treatment with ${ }^{131}$ I should be carried out, as in the protocol for metastatic thyroid cancer, before other procedures are undertaken; this approach makes $\mathrm{Tg}$ a reliable disease marker. The administration of rhTSH before the therapeutic dose of ${ }^{131}$ I may increase the therapeutic effect while avoiding the symptoms and the risks of hypothyroidism. In PTC arising in a struma ovarii, it is also important to study the common mutations of classic PTC and to correlate these with clinical and pathological features such as younger age, tumor morphology, higher probably of lymph node involvement and poor prognosis.

\section{CONFLICT OF INTEREST AND FINANCIAL DISCLOSURE}

The authors have nothing to declare.

\section{REFERENCES}

1. Checrallah A, Medlej R, Saadè C, Khayat G, Halaby G, 2001 Malignant struma ovarii: an unusual presentation. Thyroid 11: 889-892.

2. Rotman-Pikielny P, Reynolds J, Barker W, Yen P, Skarulis
M, Sarlis N, 2000 Recombinant human thyrotropin for the diagnosis and treatment of a highly functional metastatic struma ovarii. J Clin Endocrinol Metab 85: 237-244.

3. Simkin PH, Ramirez LA, Zweing SL, et al, 1999 Monomorphic teratoma of the ovary: a rare case of triiodothyronine toxicosis. Thyroid 9: 949-954.

4. Stanojievic B, Dzodic R, Saenko V, et al, 2012 Unilateral follicular variant of papillary thyroid carcinoma with unique KRAS mutation in struma ovarii in bilateral ovarian teratoma: a rare case report. BMC Cancer 12: 224-229.

5. Pardo-Mindan FJ, Vazquez JJ, 1993 Malignant struma ovarii. Light and electron microscopic study. Cancer 51: 337-343.

6. Shrimali RK, Shaikh G, Reed NS, 2012 Malignant struma ovarii: the west of Scotland experience and review of literature with focus on postoperative management. J Med Imaging Radiat Oncol 56: 478-482.

7. Jean S, Tanyi JL, Montone K, McGrath C, Martines Lage-Alvarez M, Chu CS, 2012 Papillary thyroid cancer arising in struma ovarii. J Obstetrics Gynecol 32: 222-226.

8. De Simone CP, Lele SM, Modestt SC, 2003 Malignant struma ovarii: a case report and analysis of cases reported in literature with focus on survival and 131 I therapy. Gynecol Oncol 89: 543-548.

9. Schmidt J, Derr V, Heinrich MC, et al, 2007 BRAF in papillary thyroid carcinoma of ovary (struma ovarii). Am J Surg Pathol 31: 1337-1343.

10. Xing M, 2013 BRAF V600E mutation and papillary thyroid cancer. JAMA 7: 310-535.

11. Melillo RM, Castellone MD, Guarino V, et al, 2005 The RET/PTC-RAS-BRAF linear signalling cascade mediates the motile and mitogenic phenotype of thyroid cancer cells. J Clin Invest 115: 1068-1081.

12. Romei C, Elisei R, 2012 RET/PTC Translocations and clinic-pathological features in human papillary thyroid carcinoma. Front Endocrinol 3: 54. 